\title{
基于高光谱数据的滴灌甜菜叶片全氮含量估算
}

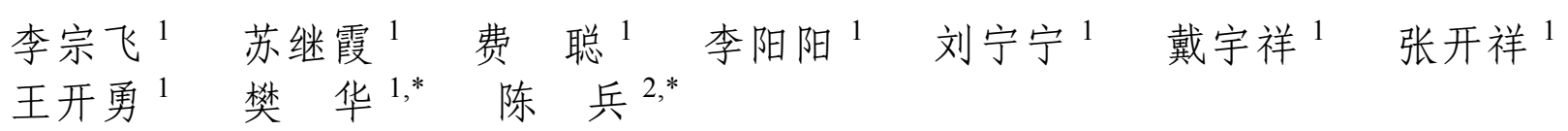

${ }^{1}$ 石河子大学农学院, 新疆石河子 $832003 ;{ }^{2}$ 新疆农圼科学院棉花研究所, 新疆石河子 832003

摘 要: 本文旨在明确甜菜叶片全氮含量与高光谱地面植被遥感的定量关系, 建立干旱区甜菜叶片全氮含量精确估 测模型, 及时监测甜菜生长状况。本研究选取新疆滴灌甜菜(Beta356)为材料, 利用 ASD 野外高光谱仪在甜菜叶丛快 速生长期、块根膨大期与糖分积累期采集各处理反射光谱, 并同时测定全氮含量, 分析原始光谱反射率及一阶微分光 谱反射率与全氮含量的相关性, 并进一步建立光谱特征参数与敏感波段植被指数全氮含量估算模型。结果表明, 光谱 特征参数 Dr762 幂函数下估算模型具有较好估算甜菜叶片全氮含量的能力, 其决定系数 $R^{2}=0.747$, 验证相对误差 $\mathrm{RE}(\%)$ 为 21.635 , 验证均方根误差 RMSE 为 4.914 ; 通过植被指数与叶片全氮含量建立多种函数估测模型, 其中差值 植被指数 Dr762-Dr496 下一元线性函数具有较好估算甜菜叶片全氮含量的能力, 其决定系数 $R^{2}=0.794$, 验证相对误 差 $\mathrm{RE}(\%)$ 为 23.008 , 验证均方根误差为 5.372 。

关键词: 全氮; 高光谱; 特征参数; 植被指数; 估算模型

\section{Estimation of total nitrogen content in sugarbeet leaves under drip irrigation based on hyperspectral characteristic parameters and vegetation index}

\author{
LI Zong-Fei ${ }^{1}$, SU Ji-Xia ${ }^{1}$, FEI Cong ${ }^{1}$, LI Yang-Yang ${ }^{1}$, LIU Ning-Ning ${ }^{1}$, DAI Yu-Xiang ${ }^{1}$, ZHANG Kai-Xiang $^{1}$, \\ WANG Kai-Yong ${ }^{1}$, FAN Hua ${ }^{1, *}$, and CHEN Bing ${ }^{2, *}$ \\ ${ }^{1}$ Agronomy College, Shihezi University, Shihezi 832003, Xinjiang, China; ${ }^{2}$ Cotton Institute, Xinjiang Academy of Agricultural and Reclamation \\ Science, Shihezi 832003, Xinjiang, China
}

\begin{abstract}
The purpose of this paper is to clarify the quantitative relationship between total nitrogen content of sugar beet and high-resolution vegetation remote sensing, to explore the establishment of an optimal estimation model for total nitrogen content of sugar beet, and to monitor the growth of sugar beet. Xinjiang drip-irrigated sugar beet (Beta356) was selected to collect the reflectance spectra of leaf cluster during the leaves rapid growth period, root expansion period and sugar accumulation period by the ASD field hyperspectral apparatus. The total nitrogen content was also measured and the relationship between original spectral reflectance and total nitrogen content was analyzed. According to the correlation between the first-order differential spectral reflectance and total nitrogen content, a total nitrogen content estimation model was established. The model with spectral characteristic parameter Dr762 power function had a good ability to estimate total nitrogen content in leaves of beet, with the determination coefficient, relative error, and root mean square error of $0.747,21.635$, and 4.914 , respectively. Various function estimation models were established based on vegetation index and leaf total nitrogen content. The linear function under vegetation index Dr762-Dr496 had better ability to estimate leaf total nitrogen content. Its determinant coefficient, relative error, and root mean square error were $0.794,23.008$, and 5.372, respectively.
\end{abstract}

Keywords: total nitrogen; hyperspectral; characteristic parameters; vegetation index; estimation model

\footnotetext{
本研究由国家自然科学基金项目(31660360，31771720), 自治区研究生科研创新项目(XJGRI2016039)和石河子大学国际科技合作推进 计划(GJHZ201706)资助。

This study was supported by the National Natural Science Foundation of China (31660360, 31771720), the Research and Innovation Projects of Postgraduates in Autonomous Region (XJGRI2016039), and the International Science and Technology Cooperation Promotion Plan of Shihezi University (GJHZ201706).

*通信作者(Corresponding authors): 樊华, E-mail: fanhua@shzu.edu.cn; 陈兵, E-mail: zyrcb@126.com

第一作者联系方式: E-mail: shzulizongfei@163.com
}

Received (收稿日期): 2019-03-20; Accepted (接受日期): 2019-12-26; Published online (网络出版日期): 2020-01-17.

URL: http://kns.cnki.net/kcms/detail/11.1809.S.20200117.1006.002.html 
氮素是甜菜生长必不可少的营养元素, 是构成 植物体内蛋白质、核酸、叶绿素以及多种酶的主要 成分, 直接反映植被营养状况, 在植物光合作用与 生长发育中发挥着重要作用 ${ }^{[1-2]}$, 因此如何根据作物 生长发育状况来判断作物适宜的追肥量, 如何运用 不同氮素运筹管理以及氮素水平来合理施肥 ${ }^{[3]}$, 快 速、无损监测甜菜生长状况, 制定适时适宜施肥方 案, 对甜菜生产具有重要意义。

传统氮素营养诊断手段以分析测试为主, 因时 效性差, 难以在大面积农业生产中有效应用 ${ }^{[4]}$ 。与传 统方法相比, 高光谱遥感可以得到作物生长发育中 生理生态与营养特征等微小特性变化, 实现对目标 实时、快速监测 ${ }^{[5]}$ 。近年来, 国内外学者研究了在不 同施氮处理条件下多种作物的光谱反射特性, 发现 了作物不同施氮处理条件下光谱反射率的差异性, 并可用具体波段与波段组合来估测氮素含量。 Shibayama 等 ${ }^{[6]}$ 在研究水稻叶片光谱特性时发现, 缺 氮水稻叶片与正常水稻叶片的光谱特征显著不同。

Smith 等 ${ }^{[7]}$ 研究发现对于同一种植物在不同时期氮 素的最佳反演波段存在变化。Stone 等 ${ }^{[8]}$ 基于 $671 \mathrm{~nm}$ 和 $780 \mathrm{~nm}$ 两个波段反射率组合的氮光谱指数对小 麦植株的全氮含量进行了估算。Thomas 等 ${ }^{[9]}$ 研究发 现 550 675 nm 波段与氮素含量具有较好相关性, 可 用来估测辣椒的氮素含量。Tian 等 ${ }^{[10]}$ 基于不同氮素 水平和多个田间试验, 提出 3 个蓝光波段构建的植 被指数 R434/(R496×R401)对叶层氮浓度具有较好预 测效果。Wang 等 ${ }^{[11]}$ 对归一化植被指数(NDVI)改进, 提出三波段指数 (R924-R703+2×R423)/(R924+ R703-2×R423), 有效提高氮素反演精度。Menesatti 等 ${ }^{[12]}$ 利用高光谱数据建立了锦橙 $\mathrm{LNC}$ 的偏最小二 乘法估算模型。Zhang 等 ${ }^{[13]}$ 建立了近红外反射光谱 与苹果 LNC 的多元线性回归方程。

在我国, 利用高光谱技术研究作物氮素营养

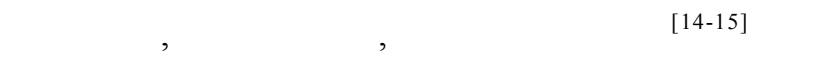
稻 ${ }^{[16-17]}$ 、棉花 ${ }^{[18-19]}$ 等作物上得到广泛应用, 赵春江 等 ${ }^{[20]}$ 对小麦不同品种、肥水条件下光谱参数变化规 律及与植株生化组分关系研究提出, 可用红边振幅 和近红外平台振幅来反演小麦叶片全氮含量。王树 文等 ${ }^{[21]}$ 采用回归分析方法对寒地水稻氮素含量建模 分析, 发现用特征光谱参数 SI (866)、DI (730、715)、 DDI (730、715、685)、DDI (866、685、558)可以较 好预测氮素含量。陈兵等 ${ }^{[22]}$ 研究了基于光谱红边参数 的棉花黄萎病叶片氮素含量诊断, 发现以 Area672 为
自变量建立的棉叶 $\mathrm{LNC}$ 的诊断模型能较好地诊断黄 萎病棉叶 $\mathrm{LNC}$ 。

尽管前人利用光谱变量估测作物农学参数方面 已经开展了大量的研究, 但在甜菜上涉及不多, 相 关甜菜氮素营养光谱诊断研究较少, 且氮素估算模 型方法较为单一。本文以滴灌甜菜为研究对象, 通 过篮选甜菜叶片全氮含量敏感波段, 构建叶片全氮 含量敏感参数与植被指数, 建立并对比叶片全氮含 量的一元线性、二次项、幂函数以及指数函数估算 模型, 以期为滴灌甜菜叶片全氮含量监测和氮肥精 准管理提供支持。

\section{1 材料与方法}

\section{1 试验设计}

试验于 2017 年 4 月至 2017 年 11 月在新疆石河 子大学农学院试验站 $\left(44^{\circ} 31^{\prime} \mathrm{N}, 88^{\circ} 06^{\prime} \mathrm{E}\right)$ 进行。试验区 域耕层土壤为灌溉灰漠土, $\mathrm{pH} 7.3$, 含有机质 $13.27 \mathrm{~g} \mathrm{~kg}^{-1}$ 、全氮 $0.76 \mathrm{mg} \mathrm{kg}$ 、速效磷 $22.56 \mathrm{mg} \mathrm{kg}^{-1}$ 、速效钾 $157.97 \mathrm{mg} \mathrm{kg}^{-1}$ 。共设置 $\mathrm{N} 0$ $\left(0 \mathrm{~kg} \mathrm{hm}^{-2}\right) 、 \mathrm{~N} 75\left(75 \mathrm{~kg} \mathrm{hm}^{-2}\right) 、 \mathrm{~N} 150\left(150 \mathrm{~kg} \mathrm{hm}^{-2}\right) 、$ $\mathrm{N} 225\left(225 \mathrm{~kg} \mathrm{hm}^{-2}\right) 4$ 个施氮水平。施氮比例按照 M0 (0：0：0)、M1 (10：0：0)、M2 (7：3：0)、M3 (5：3：2) 3 种运筹模式, 即分别在甜菜叶丛快速生 长期、块根膨大期与块根糖分积累期按比例施入氮 肥。采用随机区组设计, 重复 3 次, 供试甜菜品种为 Beta356, 播种日期为 4 月 15 日, 试验田内设置 30 个小区, 小区面积 $12 \mathrm{~m}^{2}$, 甜菜播种行距 $50 \mathrm{~cm}$, 株

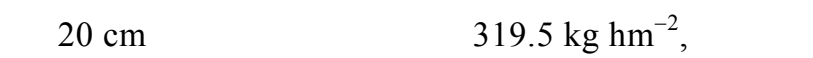
$206 \mathrm{~kg} \mathrm{hm}^{-2}$ 。其他按照大田统一管理。

\section{2 甜菜冠层光谱数据测定}

采用美国 Analytical Spectral Device (ASD)公司 生产的 FileSpecPro FR2500型背挂式野外高光谱辐射 仪测定甜菜植株冠层高光谱反射率。该光谱仪波段范 围为 350 2500 nm, 采样间隔为 $1 \mathrm{~nm}$, 光谱仪视场角 $25^{\circ}$, 测量时探头垂直向下, 距离冠层垂直高度约 $0.5 \mathrm{~m}$, 在测量前均进行白板校正。选择晴朗无云天气, 于当地北京时间 12:00-15:00 分别在甜菜叶丛快速 生长期、块根膨大期、糖分积累期测定光谱反射率, 记录每个光谱测定点 5 条光谱数据, 每小区测定 3 次, 取其平均值作为该小区的光谱反射率值。

\section{3 全氮含量测定}

采用凯氏定氮法测定叶片全氮含量。取光谱测 定区域叶片, $105^{\circ} \mathrm{C}$ 杀青 $30 \mathrm{~min}$ 后, 调至 $75^{\circ} \mathrm{C}$ 烘干至 
恒重, 将烘干样品粉碎过 60 目篮。用分析天平秤取 $0.5000 \mathrm{~g}$ 叶片样品放入消煮管, 采用 $\mathrm{H}_{2} \mathrm{O}_{2}$-浓 $\mathrm{H}_{2} \mathrm{SO}_{4}$ 消化处理后, 将冷却的消煮液置于定氮仪中蒸馏, 利用 $0.01 \mathrm{~mol} \mathrm{~L}^{-1}\left(1 / 2 \mathrm{H}_{2} \mathrm{SO}_{4}\right)$ 标准溶液滴定馏出液由 蓝绿色至刚变为紫红色, 记录所用标准酸体积, 根 据以下公式计算植株全氮含量。

叶片全氮 $(\mathrm{N})$ 量 $\left(\mathrm{g} \mathrm{kg}^{-1}\right)=\frac{C \times\left(V-V_{0}\right) \times 0.014 \times T_{\mathrm{S}} \times 100}{m}$ 式中, $C$ 是 $0.01 \mathrm{~mol} \mathrm{~L}^{-1}\left(1 / 2 \mathrm{H}_{2} \mathrm{SO}_{4}\right)$ 标准溶液浓度, $V$ 和 $V_{0}$ 分别是滴定试液时所用酸标准溶液的体积与滴 定空白时所用酸标准溶液的体积 $(\mathrm{mL}), 0.014$ 为氮的 摩尔质量 $\mathrm{kg} \mathrm{mol}^{-1}, T_{\mathrm{S}}$ 为分取倍数, 即定容体积与分 取体积之比, 1000 为 $\mathrm{kg}$ 与 $\mathrm{g}$ 之间的换算倍数, $m$ 为
烘干株样的质量 $(\mathrm{g})$ 。

\section{4 数据分析}

光谱原数据提取和预处理采用 FileSpecPro FR2500 型背挂式野外高光谱辐射仪自带软件 ViewSpec Program，用 Microsoft Excel 2013 与 SPSS 12.0 软件统计分析农学参数数据和光谱数据, 并利 用 Origin 2018 软件制图, 采用 MATLAB 软件提取甜 菜冠层光谱数据的特征参数, 从中提取常用光谱特 征“三边”参数; 通过选择已有光谱植被指数作对比, 结合与本试验中全氮含量相关性较好的敏感性波段, 构建比值 (RVI)、差值(DVI)、归一化比值(NDVI)与 红边归一化差异(NDI)光谱植被指数(表 1)。

\section{表 1 高光谱特征参数及植被指数定义}

Table 1 Hyperspectral parameters and their definitions

\begin{tabular}{|c|c|}
\hline $\begin{array}{c}\text { 光谱特征参数与植被指数 } \\
\text { Spectral characteristic parameter and Spectral index }\end{array}$ & $\begin{array}{c}\text { 定义 } \\
\text { Definition }\end{array}$ \\
\hline 红边斜率 & 680-760 nm 内最大一阶微分光谱值 \\
\hline Red edge slope (Dr) & Maximum first order differential spectrum in $680-760 \mathrm{~nm}$ \\
\hline 红边位置 & 680-760 nm 内最大的一阶微分光谱值对应的波长 \\
\hline Red edge position $(\lambda r)$ & $\begin{array}{l}\text { The wavelength corresponding to the largest first-order differential spectrum in } \\
680-760 \mathrm{~nm}\end{array}$ \\
\hline 红边面积 & 680-760 nm 内一阶微分光谱值的总和 \\
\hline Red edge area (SDr) & The sum of the first-order differential spectral values in $680-760 \mathrm{~nm}$ \\
\hline 黄边斜率 & 560-640 nm 内最大一阶微分光谱值 \\
\hline Yellow edge slope (Dy) & Maximum first order differential spectrum in $560-640 \mathrm{~nm}$ \\
\hline 黄边位置 & 560-640 nm 内最大一阶微分光谱值对应的波长 \\
\hline Yellow edge position $(\Lambda y)$ & $\begin{array}{l}\text { The wavelength corresponding to the largest first-order differential spectrum in } \\
560-640 \mathrm{~nm}\end{array}$ \\
\hline 黄边面积 & 560-640 nm 内一阶微分光谱值的总和 \\
\hline Yellow edge area (SDy) & The sum of the first-order differential spectral values in $560-640 \mathrm{~nm}$ \\
\hline 蓝边斜率 & 490-530 nm 内最大一阶微分光谱值 \\
\hline Blue edge slope (Db) & Maximum first order differential spectrum in $490-530 \mathrm{~nm}$ \\
\hline 蓝边位置 & 490-530 nm 内最大一阶微分光谱值对应的波长 \\
\hline Blue edge position $(\lambda b)$ & $\begin{array}{l}\text { The wavelength corresponding to the largest first-order differential spectrum in } \\
490-530 \mathrm{~nm}\end{array}$ \\
\hline 蓝边面积 & 490-530 nm 内一阶微分光谱值的总和 \\
\hline Blue edge area $(\mathrm{SDb})$ & The sum of the first-order differential spectral values in $490-530 \mathrm{~nm}$ \\
\hline $\begin{array}{l}\text { 比值植被指数 } \\
\text { Ratio vegetation index (RVI) }\end{array}$ & $\mathrm{R}_{\lambda 1} / \mathrm{R}_{\lambda 2}$ \\
\hline $\begin{array}{l}\text { 差值植被指数 } \\
\text { Difference vegetation index (DVI) }\end{array}$ & $\mathrm{R}_{\lambda 1}-\mathrm{R}_{\lambda 2}$ \\
\hline $\begin{array}{l}\text { 归一化比值植被指数 } \\
\text { Normalized ratio vegetation index (NDVI) }\end{array}$ & $\left(\mathrm{R}_{\mathrm{NIR}}-\mathrm{R}_{\mathrm{red}}\right) /\left(\mathrm{R}_{\mathrm{NIR}}+\mathrm{R}_{\mathrm{red}}\right)$ \\
\hline $\begin{array}{l}\text { 红边归一化差异指数 } \\
\text { Red edge normalized difference index (NDI) }\end{array}$ & $\left(\mathrm{DR}_{\lambda 1}-\mathrm{DR}_{\lambda 2}\right) /\left(\mathrm{DR}_{\lambda 1}+\mathrm{DR}_{\lambda 2}\right)$ \\
\hline
\end{tabular}

$R_{\lambda 1}$ 与 $R_{\lambda 2}$ 分别为 $\lambda_{1}$ 与 $\lambda_{2}$ 波长处的光谱反射率。

$R_{\lambda 1}$ and $R_{\lambda 2}$ in the equation are the spectral reflectance at wavelengths $\lambda_{1}$ and $\lambda_{2}$. 
通过分析光谱特征参数及植被指数与甜菜冠层 叶片全氮含量相关性, 建立全氮含量估测模型。本 文主要针对甜菜叶丛快速生长期至糖分积累期 3 个 时期进行测定, 甜菜在叶丛快速生长期后基本达到 土壤背景全覆盖, 且甜菜自叶丛快速生长期至糖分 积累期冠层结构无明显变化, 因此本文选择总样本 量用于甜菜叶片全氮含量估算与检验, 根据测定样 本数量大小, 将采集样本随机分为 2 组, 其中建模 样本 75 个, 模型检验样本 26 个, 通过对比各模型 的建模决定系数 $\left(R^{2}\right)$ 、均方根误差( RMSE) 以及相对 误差 $(\mathrm{RE})$ 䇻选出最优模型用以研究新疆滴灌条件 下甜菜冠层叶片全氮含量的估算。其中 $R^{2}$ 用以判 断模型拟合优度, RE 与 RMSE 用于检验估测模型 的可靠程度。

$$
\begin{aligned}
& \mathrm{RE}=\frac{1}{n} \sum_{i=1}^{n} \frac{\left|\hat{y}_{i}-y_{i}\right|}{y_{i}} \times 100 \% \\
& \mathrm{RMSE}=\sqrt{\frac{1}{n} \sum_{i=1}^{n}\left(\hat{y}_{i}-y_{i}\right)^{2}}
\end{aligned}
$$

式中, $y_{i}$ 为试验测定全氮含量真实值, $\hat{y}_{i}$ 为模型估测 全氮含量估测值, $n$ 为样本数量。

\section{2 结果与分析}

2.1 不同氮素水平下甜菜冠层光谱特征分析 通过对不同氮素水平下光谱数据进行平均处理, 由原始光谱曲线图与一阶导数光谱曲线图分析可知
(图 1), 不同氮素水平下甜菜原始冠层光谱反射率波 形趋势相似，在波段 350 670 nm 范围内有明显的反 射峰与吸收谷, 光谱波形呈现先增高后降低的趋势, 在 $550 \mathrm{~nm}$ 附近形成反射小高峰, 均在 $670 \mathrm{~nm}$ 附近 形成吸收谷, 在波段 670 760 nm 波段波形变化较为 剧烈, 光谱反射率随着波长的增加急剧上升, 形成 较大陡坡状波形; 在近红外波段 780 1300 nm 形成 近红外反射平台, 且具有明显的波形变化。施氮处 理 N75、N150、N225 在 $550 \mathrm{~nm}$ 处较 CK 分别降低 了 $7.5 \% 、 28.0 \% 、 23.0 \%$, 说明各施氮处理相对 $\mathrm{CK}$ 均可提高甜菜植株冠层叶片对红、蓝光的吸收, 提 高植株的光合效率, 且施氮处理 N150 比 N75 与 N225 具有更好地提高植株光合效率的作用; 在近红 外波段(780 1300 nm)各施氮处理较 $\mathrm{CK}$ 分别增加 $6.14 \% \sim 12.6 \% 、 14.2 \% \sim 16.4 \% 、 6.82 \% \sim 18.0 \%$, 各处 理之间较容易区分。

由图 1 可知, 红边与原始冠层光谱图曲线近红外 (780 1300 nm)波段基本一致，各施氮处理之间有明 显差异, 且具有“双峰”现象, 说明不同施氮处理对甜 菜植株有较大影响, 不同施氮处理除 N75 红边位置 与 N0 相同, 其他施氮处理均出现“红移”现象, 施氮 处理 N150、N225 较 N0 均向长波方向移动 $5 \mathrm{~nm}$, 说 明随着施氮量的增加, 甜菜植株冠层单位光合效率 有所提高, 但在施氮处理 N150 后红边位置趋于平稳, 这与原始光谱反射率可见光波段“绿峰”处保持一致。
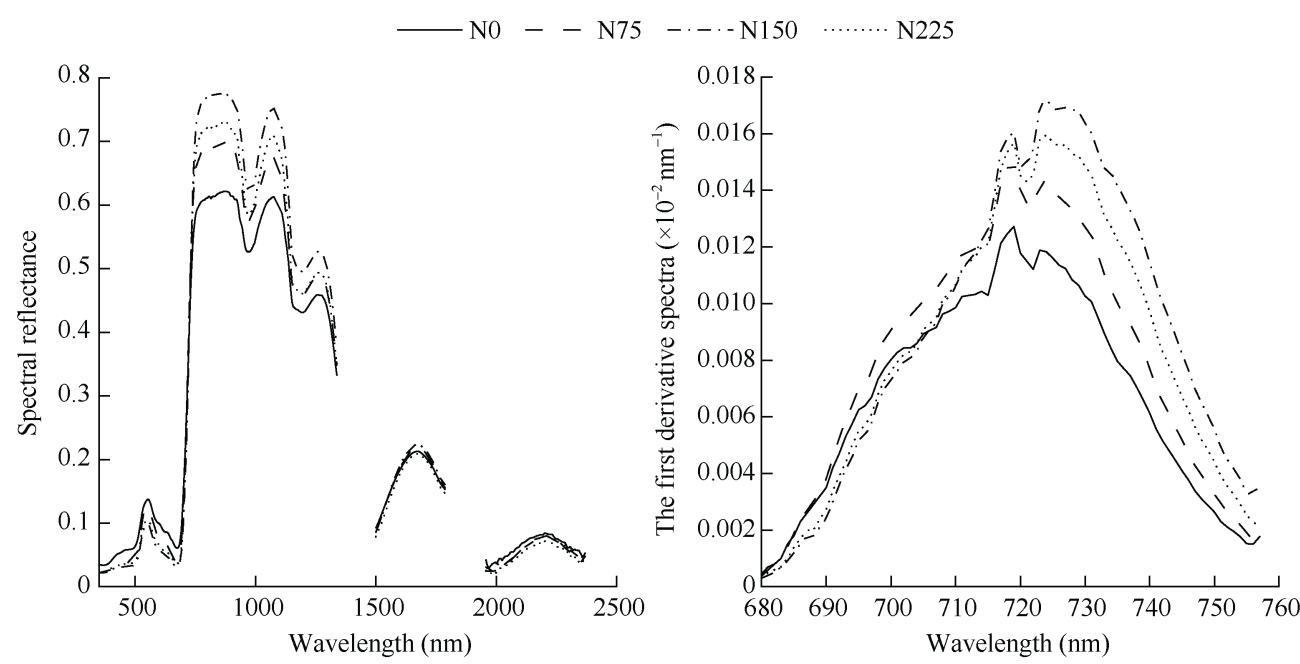

图 1 不同氮素水平下高光谱反射率与红边

Fig. 1 Canopy hyperspectral reflectance and red edge at different nitrogen levels

N0: 施氮 $0 \mathrm{~kg} \mathrm{hm}^{-2}$; N75: 施氮 $75 \mathrm{~kg} \mathrm{hm}^{-2}$; N150: 施氮 $150 \mathrm{~kg} \mathrm{hm}^{-2}$; 225 : 施氮 $225 \mathrm{~kg} \mathrm{hm}^{-2}$ 。

$\mathrm{N} 0$ : nitrogen application of $0 \mathrm{~kg} \mathrm{hm}^{-2}$; N75: nitrogen application of $75 \mathrm{~kg} \mathrm{hm}^{-2}$; N150: nitrogen application of $150 \mathrm{~kg} \mathrm{hm}{ }^{-2}$; N225: nitrogen application of $225 \mathrm{~kg} \mathrm{hm}^{-2}$. 


\section{2 原始光谱反射率与全氮含量的相关性}

结果如图 2 所示, 冠层光谱反射率与冠层叶片 相关性稳定波段主要集中 350 1300 nm 与 1418 $1809 \mathrm{~nm}$, 由于在 1300 2500 $\mathrm{nm}$ 叶面光谱反射率主 要受叶内水含量的控制, 叶内水含量降低, 其光谱 反射率升高, 且氮素含量的敏感波段主要集中在短 波红外与可见光波段, 所以选择 350 1300 $\mathrm{nm}$ 作为 全氮含量估测波段。

由图 2 可知, 在 350 1300 $\mathrm{nm}$ 范围内, 在可见光
波段与冠层叶片全氮含量呈现极显著正相关的波段 主要集中在 726 780 nm, 且在波段 $767 \mathrm{~nm}$ 处达到最 大正相关 $(r=0.609, P<0.01)$, 与全氮含量呈现极显 著负相关的波段主要集中在 591 615 nm，且在波段 $604 \mathrm{~nm}$ 达到最大负相关 $(r=-0.267, P<0.01)$; 在近 红外波段与冠层叶片全氮含量呈现极显著正相关的 波段主要集中在 780 1300 nm，且在波段 $1104 \mathrm{~nm}$ 处 达到最大正相关 $(r=0.68, P<0.01)$, 但在此波段范围 内没有出现与全氮含量的极显著负相关。

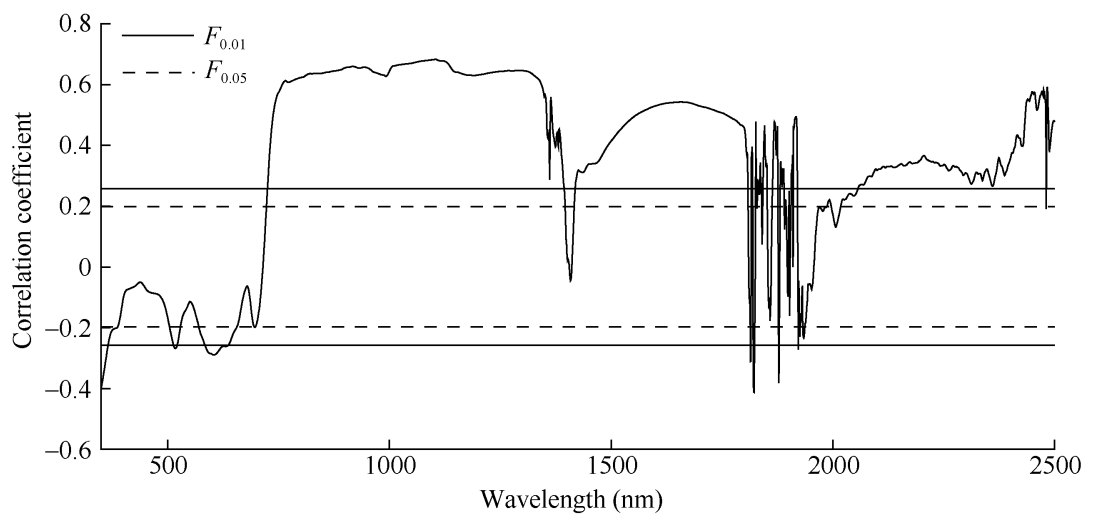

图 2 甜菜冠层原始光谱反射率与全氮含量相关性

Fig. 2 Correlation between original spectral reflectance and total nitrogen content in sugar beet canopy $F_{0.05}$ ：显著相关 $(P<0.05) ; F_{0.01}$ ：极显著相关 $(P<0.01)$ 。

$F_{0.05}$ : significant correlation $(P<0.05) ; F_{0.01}$ : extremely significant correlation $(P<0.01)$.

\section{3 一阶微分光谱与全氮含量相关性}

叶片全氮含量的敏感波段主要集中在短波红外 与可见光波段，因此选择 350 1300 nm 一阶导数光 谱反射率与叶片全氮含量作相关性分析。由图 3 可 知，在波段 389 403、610 620、638 679、709 766、 774 805、995 1073、1200 1252 nm 范围内呈现极 显著正相关，且在可见光 $(380 \sim 780 \mathrm{~nm})$ 范围内在波 长 $762 \mathrm{~nm}$ 处达到最大正相关 $(r=0.703, F<0.01)$, 在
近红外(780 1300 nm)范围内在波长 $1018 \mathrm{~nm}$ 处达到 最大正相关 $(r=0.633, F<0.01)$; 在波段 445 457、 486 517、683 693、943 964、1126 1176、1285 1300 $\mathrm{nm}$ 范围内呈现极显著负相关关系，且在可见光 (380 780 nm)范围内在波长 $496 \mathrm{~nm}$ 处达到最大负相 关 $(r=-0.545, F<0.01)$, 在近红外波段(780 1300 nm) 范围内在波长 $1138 \mathrm{~nm}$ 处达到最大负相关 $(r=$ $-0.715, F<0.01)$; 在红边 $(680 \sim 760 \mathrm{~nm})$ 范围内, 最大

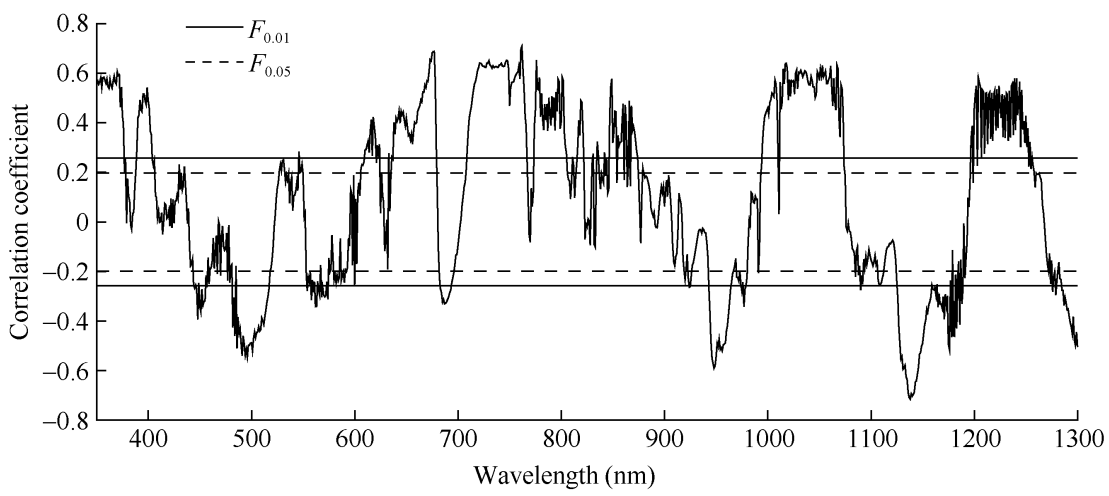

图 3 甜菜冠层一阶导数光谱与全氮含量相关性

Fig. 3 Correlation between the first derivative spectra of sugar beet canopy and the total nitrogen content $F_{0.05}$ ：显著相关 $(P<0.05) ; F_{0.01}$ ：极显著相关 $(P<0.01)$ 。

$F_{0.05}$ : significant correlation $(P<0.05) ; F_{0.01}$ : extremely significant correlation $(P<0.01)$. 
正相关位于波长 $747 \mathrm{~nm}$ 处 $(r=0.646, F<0.01)$, 最大 负相关位于波长 $687 \mathrm{~nm}$ 处 $(r=-0.332, F<0.01)$ 。

2.4 光谱特征参数、敏感波段植被指数与全氮含 量相关性

全氮含量与一阶导数光谱的相关性，在相应波段
内选取相关系数最大的对应波段反射率, 构建原始光 谱反射率的比值、差值与归一化比值植被指数, 构建 一阶微分光谱反射率的比值、差值、归一化比值与红 边比值、差值与归一化差异指数。研究并分析三边参 数及敏感波段植被指数与全氮含量的相关性(表 2)。

表 2 光谱特征参数及敏感波段植被指数与全氮含量相关性

Table 2 Correlation between spectral characteristic parameters and vegetation index in sensitive bands and total nitrogen content

\begin{tabular}{|c|c|c|c|}
\hline $\begin{array}{c}\text { 光谱特征参数 } \\
\text { Spectral characteristic } \\
\text { parameter }\end{array}$ & $\begin{array}{l}\text { 相关关系 } \\
\text { Correlation }\end{array}$ & $\begin{array}{c}\text { 植被指数 } \\
\text { Vegetation index }\end{array}$ & $\begin{array}{c}\text { 相关性 } \\
\text { Correlation }\end{array}$ \\
\hline $\operatorname{Dr}$ & $0.590^{* *}$ & 原始光谱比值植被指数 Ratio vegetation index R1104/R767 & $0.358^{* *}$ \\
\hline$\lambda \mathrm{r}$ & $0.497^{* *}$ & 原始光谱差值植被指数 Difference vegetation index R1104-R767 & $0.280^{* *}$ \\
\hline SDr & $0.601^{* *}$ & $\begin{array}{l}\text { 原始光谱归一化植被指数 Normalized ratio vegetation index } \\
(\mathrm{R} 1104-\mathrm{R} 767) /(\mathrm{R} 1104+\mathrm{R} 767)\end{array}$ & $0.364^{* *}$ \\
\hline Dy & 0.074 & 原始光谱比值植被指数 Ratio vegetation index R767/R604 & $0.652^{* *}$ \\
\hline$\Lambda y$ & 0.089 & 原始光谱差值植被指数 Difference vegetation index R767-R604 & $0.433^{* *}$ \\
\hline SDy & -0.086 & 一阶微分比值植被指数 Ratio vegetation index Dr1138/Dr762 & -0.169 \\
\hline $\mathrm{Db}$ & 0.072 & 一阶微分差值植被指数 Difference vegetation index Dr1138-Dr762 & $-0.744^{* *}$ \\
\hline$\lambda \mathrm{b}$ & $0.506^{* *}$ & $\begin{array}{l}\text { 一阶微分归一化植被指 Normalized ratio vegetation index } \\
\text { (Dr1138-Dr762)/(Dr1138+Dr762) }\end{array}$ & $0.500^{* *}$ \\
\hline $\mathrm{SDb}$ & -0.216 & 一阶微分比值植被指数 Ratio vegetation index Dr762/Dr496 & $0.586^{* *}$ \\
\hline R604 & $-0.267^{* *}$ & 一阶微分差值植被指数 Difference vegetation index Dr762-Dr496 & $0.721^{* *}$ \\
\hline R1104 & $0.680^{* *}$ & 红边比值植被指数 Red edge ratio vegetation index $\operatorname{Dr} 747 / \operatorname{Dr} 687$ & $0.431^{* *}$ \\
\hline Dr1138 & $-0.715^{* *}$ & 红边差值植被指数 Red edge difference vegetation index Dr747-Dr687 & $0.589^{* *}$ \\
\hline Dr762 & $0.703^{* *}$ & $\begin{array}{l}\text { 红边归一化差异指数 Red edge normalized difference index } \\
(\text { Dr747-Dr687)/( Dr747+Dr687) }\end{array}$ & $0.545^{* *}$ \\
\hline
\end{tabular}

${ }^{* *}$ 极显著水平 $(P<0.01)$ 。 R604: 原始光谱最大负相关; R1104：原始光谱最大正相关; Dr1138：一阶微分最大负相关; Dr762: 一阶微分 最大正相关。缩写同表 1 。

** means the significance at the 0.01 probability level. R604: maximum negative correlation of original spectrum; R1104: maximum positive correlation of original spectrum; Dr1138: maximum negative correlation of first order differential; Dr762: maximum positive correlation of first order differential. Abbreviations are the same as those given in Table 1.

由表 2 可知, 所选光谱特征参数中红边参数与 叶片全氮含量均达到极显著相关, 黄边参数与蓝边 参数中只有蓝边位置与叶片全氮含量达到极显著相 关, 在三边参数中, 红边振幅、红边面积、蓝边位置 与叶片全氮含量均达到 0.5 以上的极显著相关, 其 中红边面积与之达到最大相关 $(r=0.601, F<0.01)$; 原始光谱反射率和一阶微分光谱反射率与叶片全氮 含量的最大相关性均达到极显著水平, 其中一阶微 分最大负相关 Dr1138 与一阶微分最大正相关 Dr762 的相关性均达到 0.7 以上; 所建植被指数与全氮含 量的相关性除一阶微分比值植被指数 Dr1138/Dr762 没有达到极显著水平, 其他植被指数均达到极显著 水平, 但整体与叶片全氮含量的相关性较小, 在原 始光谱植被指数中, 只有原始比值植被指数 R767/ R604 与叶片全氮含量的相关性达到了 0.6 以上; 在
一阶微分植被指数与叶片全氮含量的相关性中, 除 一阶微分比值植被指数 Dr1138/Dr762 外均达到 0.5 以上极显著水平, 其中一阶微分差值植被指数 Dr1138-Dr762 与一阶微分差值植被指数 Dr762Dr496 的相关系数均达到 0.7 以上; 红边植被指数与 叶片全氮含量均达到极显著相关水平, 其中红边差 值植被指数 Dr747-Dr687 与红边归一化差异指数的 均达到 0.5 以上, 且一阶微分光谱植被指数和红边 光谱植被指数与叶片全氮含量的相关整体大于原始 光谱植被指数的相关性。

\section{5 已知高光谱植被指数与全氮含量相关性}

在前人研究的基础上, 针对本试验数据对已有 植被指数优选, 分析优选高光谱植被指数与全氮含 量的相关性, 由表 3 可知, 所选的 15 个高光谱植被 指数均与全氮含量极显著相关, 其中达到 0.5 以上 
相关性的有 8 个，植被指数 SAVI、OSAVI 和 MSAVI 植被指数 SAVI 与全氮含量达到最大相关性 $(r=$ 与全氮含量的相关性均达到 0.6 以上, 且土壤调节 $\left.0.692^{* *}, F<0.01\right)$ 。

\section{表 3 已知高光谱植被指数与全氮含量的相关性}

Table 3 Correlation between known hyperspectral vegetation index and total nitrogen content

\begin{tabular}{|c|c|c|c|}
\hline $\begin{array}{c}\text { 植被指数 } \\
\text { Vegetation index }\end{array}$ & $\begin{array}{c}\text { 计算公式 } \\
\text { Calculation formula } \\
\end{array}$ & $\begin{array}{l}\text { 相关关系 } \\
\text { Correlation } \\
\end{array}$ & $\begin{array}{c}\text { 文献来源 } \\
\text { Source of literature }\end{array}$ \\
\hline RSI & R990/R720 & $0.528^{* *}$ & [23] \\
\hline NDSI & $(\mathrm{R} 860-\mathrm{R} 720) /(\mathrm{R} 860+\mathrm{R} 720)$ & $0.582^{* *}$ & [23] \\
\hline NDVI & $(\mathrm{R} 790-\mathrm{R} 670) /(\mathrm{R} 790+\mathrm{R} 670)$ & $0.332^{* *}$ & [24] \\
\hline FD-NDVI & $(\mathrm{R} 730-\mathrm{R} 525) /(\mathrm{R} 730+\mathrm{R} 525)$ & $0.451^{* *}$ & {$[25]$} \\
\hline RENDVI & (R750-R705/( R750+R705) & $0.367^{* *}$ & [26] \\
\hline mND705 & $(\mathrm{R} 750-\mathrm{R} 705) /(\mathrm{R} 750+2 \mathrm{R} 445)$ & $0.360^{* *}$ & [27] \\
\hline GNDVI & (R790-R550)/(R790+R550) & $0.464^{* *}$ & [28] \\
\hline SAVI & $1.5(\mathrm{R} 870-\mathrm{R} 680) /(\mathrm{R} 870+\mathrm{R} 680+0.5)$ & $0.692^{* *}$ & [29] \\
\hline OSAVI & $(1+0.16)(\mathrm{R} 810-\mathrm{R} 680) /(\mathrm{R} 810-\mathrm{R} 680+0.16)$ & $0.633^{* *}$ & [30] \\
\hline MSAVI & $0.5\{2 \mathrm{R} 800+1-[(2 \mathrm{R} 800+1) 2-8(\mathrm{R} 800-\mathrm{R} 670)] 0.5\}$ & $0.623^{* *}$ & {$[31]$} \\
\hline DCNI & $(\mathrm{R} 720-\mathrm{R} 700 /(\mathrm{R} 700-\mathrm{R} 670) /(\mathrm{R} 720-\mathrm{R} 670+0.03)$ & $0.460^{* *}$ & [32] \\
\hline CIgreen & {$[(\mathrm{R} 840-\mathrm{R} 870) / \mathrm{R} 550]-1$} & $-0.493^{* *}$ & [33] \\
\hline NINI & {$[\lg (1 / \mathrm{R} 1510)-\lg (1 / \mathrm{R} 1680)] /[\lg (1 / \mathrm{R} 1510)+\lg (1 / \mathrm{R} 1680)]$} & $0.553^{* *}$ & [34] \\
\hline TVI & $0.5[120(\mathrm{R} 750-\mathrm{R} 550)-200(\mathrm{R} 670-\mathrm{R} 550)]$ & $0.538^{* *}$ & {$[35]$} \\
\hline DSI & $\mathrm{R} 800-\mathrm{R} 680$ & $0.510^{* *}$ & [36] \\
\hline
\end{tabular}

*** 极显著水平 $(P<0.01)$ 。RSI：比值光谱指数；NDSI：归一化光谱指数；NDVI：归一化植被指数；FD-NDVI：微分归一化氮指数; RENDVI: 红边归一化指数; mND705: 修正红边归一化指数; GNDVI: 绿波段归一化植被指数; SAVI: 土壤调节植被指数; OSAVI: 优 化土壤调节植被指数; MSAVI：修正土壤调节植被指数; DCNI：双峰冠层氮指数; CIgreen: 绿色色素指数; NDNI: 归一化氮素指数; TVI: 三角植被指数; DSI: 差值植被指数。

${ }^{* *}$ means the significance at the 0.01 probability level. RSI: ratio spectral index; NDSI: normalized difference spectral index; NDVI: normalized difference vegetation index; FD-NDVI: normalized difference of nitrogen index; RENDVI: red edge normalized difference vegetation index; mND705: modified red-edge normalized difference vegetation index; GNDVI: green normalized difference vegetation index; SAVI: soil-adjusted vegetation index; OSAVI: optimization of soil-adjusted vegetation index; MSAVI: modified soil adjusted vegetation index; DCNI: double-peak canopy nitrogen index; CIgreen: green chlorophyll index; NDNI: normalized difference nitrogen index; TVI: triangle vegetation index; DSI: difference vegetation index.

\section{6 基于高光谱特征参数与敏感植被指数的全 氮含量的估算与验证}

选取表 2、表 3 相关系数绝对值大于 0.65 的植 被指数用于甜菜叶片全氮含量的估算。其中光谱特 征参数与植被指数作为自变量 $(x)$, 叶片全氮含量 作为因变量 $(y)$, 构建叶片全氮含量的一元线性、二 次项、幂函数以及指数函数估算模型。通过对比各 模型的建模决定系数 $\left(R^{2}\right)$ 、相对误差 $(\mathrm{RE})$ 以及均方 根误差(RMSE)篮选出最优模型用以研究新疆滴灌 条件下甜菜叶片全氮含量的估算。其中 $R^{2}$ 用以判 断模型拟合优度, RE 与 RMSE 用于检验估测模型 的可靠程度。

2.6.1 基于高光谱特征参数的全氮含量的估算与验 证通过对构建光谱特征参数模型与精度检验分
析可知(表 4), 所选光谱特征参数与全氮含量均具有 较好的拟合关系，同时具有较好的拟合精度 $\left(R^{2}=\right.$ 0.49 0.78)。通过比较不同函数估算模型发现, 不同 光谱特征参数所建模型均表现为二次项函数模型最 优, 其中光谱特征参数 Dr1138 与 Dr762 的决定系数 均达到 0.6 以上，说明在光谱短波近红外波段与红 光处参数有较好估算全氮含量的能力。通过比较决 定系数发现, 光谱特征参数 Dr762 在所选参数中表 现最优, 不同函数构建估算模型决定系数均达到 0.7 以上, 且以二次项函数所建模型决定系数最高 $\left(R^{2}=\right.$ $0.772)$, 其次为线性函数 $\left(R^{2}=0.770\right)$ 。

利用检验样本对上述变量建立的估算模型预测 精度进行检验, 各模型检验结果如表 4 所示, 通过 对比光谱特征参数 Dr762 的 RE(\%)与 RMSE 发现, 
表 4 不同光谱特征参数与全氮含量回归关系模型及验证

Table 4 Regression relationship model and verification of different spectral characteristic parameters and total nitrogen content

\begin{tabular}{|c|c|c|c|c|}
\hline \multirow{2}{*}{$\begin{array}{c}\text { 高光谱特征参数 } \\
\text { Characteristic parameter }\end{array}$} & \multicolumn{2}{|c|}{ 拟合模型 Fitting model } & \multicolumn{2}{|c|}{ 验证模型 Performance model } \\
\hline & 拟合方程 Equation & $R^{2}$ & $\mathrm{RE}(\%)$ & RMSE \\
\hline \multirow[t]{4}{*}{ R1104 } & $y=63.88 x-8.167$ & 0.516 & 21.429 & 4.762 \\
\hline & $y=-30.27 x^{2}+96.39-16.64$ & 0.519 & 22.299 & 4.829 \\
\hline & $y=58.24 x^{1.296}$ & 0.513 & 20.898 & 4.766 \\
\hline & $y=7.555 \mathrm{e}^{2.277 x}$ & 0.493 & 22.904 & 4.898 \\
\hline \multirow[t]{4}{*}{ Dr1138 } & $y=64.73 x-1.144$ & 0.643 & 19.893 & 4.874 \\
\hline & $y=-47.08 x^{2}+104.7 x-9.162$ & 0.648 & 20.625 & 4.945 \\
\hline & $y=63.68 x^{1.031}$ & 0.643 & 19.909 & 4.881 \\
\hline & $y=9.629 \mathrm{e}^{2.313 x}$ & 0.615 & 23.265 & 5.010 \\
\hline \multirow[t]{4}{*}{ Dr762 } & $y=157.8 x-147$ & 0.770 & 20.877 & 5.038 \\
\hline & $y=-192.3 x^{2}+578.8 x-377.1$ & 0.772 & 21.518 & 5.137 \\
\hline & $y=13.96 x^{6.461}$ & 0.747 & 21.635 & 4.914 \\
\hline & $y=0.04302 \mathrm{e}^{5.813 x}$ & 0.740 & 22.103 & 4.928 \\
\hline
\end{tabular}

$R^{2}$ : 决定系数; $\mathrm{RE}(\%)$ : 相对误差; RMSE: 均方根误差。缩写同表 2。

$R^{2}$ : coefficient of determination; $\mathrm{RE}(\%)$ : relative error; RMSE: relative root mean-squared error. Abbreviations are the same as those given in Table 2 .

虽然二次项函数估算模型决定系数具有更好优势, 但 RE $(\%)$ 与 RMSE 较高, 且幂函数与指数函数在验 证模型中均表现出较低的 RE(\%)与 RMSE，难以判 定出最优估算模型，因此对估测值与真实值拟合模 型再次验证, 通过对光谱特征参数 Dr762 下不同函 数估测值与真实值拟合分析发现(图 4), 幂函数估算 模型较其他估算模型具有更高的决定系数, 因此光 谱特征参数 Dr762 下幂函数估算模型具有更好的拟 合优度。

2.6.2 基于高光谱植被指数的叶片全氮含量估算与 验证通过对构建光谱植被指数与精度检验分析 可知(表 5), 所选光谱植被指数与全氮含量均具有较 好的拟合关系, 同时具有较好拟合精度 $\left(R^{2}=\right.$ $0.415 ~ 0.804)$ 。通过比较不同函数估算模型决定系数 发现, 不同植被指数所建模型均表现为二次项函数 估算模型最优，其中差值植被指数 Dr1138-Dr762 与 Dr762-Dr496 决定系数均达到 0.7 以上, 且在差值植 被指数 Dr762-Dr496 下二次项估算模型的决定系数 达到最高值 $\left(R^{2}=0.804\right)$, 这说明在短波近红外波段 与红光波段组合和红光波段与绿光波段组合具有较 好估算叶片全氮含量的能力。

利用检验样本对上述变量建立的估算模型预测
精度进行检验，各模型检验结果如表 5 所示，通过 对比差值植被指数 Dr762-Dr496 的 RE(\%)与 RMSE 发现, 差值植被指数 Dr762-Dr496 估算模型与光谱 特征参数 Dr762 表现出相似特征, 虽然二次项函数 估算模型决定系数最高, 表现出较高拟合优度, 但 $R M S E$ 在整体函数估算模型中表现最高, 说明偏离 真值的程度较大，虽然差值植被指数 Dr1138-Dr762 较 Dr762-Dr496 的决定系数有所降低, 但其表现出 较低的 RE(\%)与较低 RMSE, 因此难以对最优全氮 估算模型进行判定，因此对估测值与真实值拟合模 型再次验证, 通过对差值植被指数 Dr1138-Dr762 与 Dr762-Dr496 下不同函数估测值与真实值拟合分析 发现(图 5 和图 6), 在差值植被指数 Dr1138-Dr762 中指数函数估测值与真实值拟合程度表现最优 $\left(R^{2}=\right.$ $0.739)$, 但与差值植被指数 Dr762-Dr496 的拟合程 度相比表现较低。在差值植被指数 Dr762-Dr496 下线性函数估测值与真实值拟合决定系数和指数 函数估测值与真实值拟合决定系数相同 $\left(R^{2}=\right.$ $0.759)$, 但线性函数估算模型较指数函数估算模 型具有较高决定系数与较低 $\mathrm{RE}(\%)$, 因此差值植 被指数 Dr762-Dr496 下线性函数估算模型具有更 好的拟合优度。 

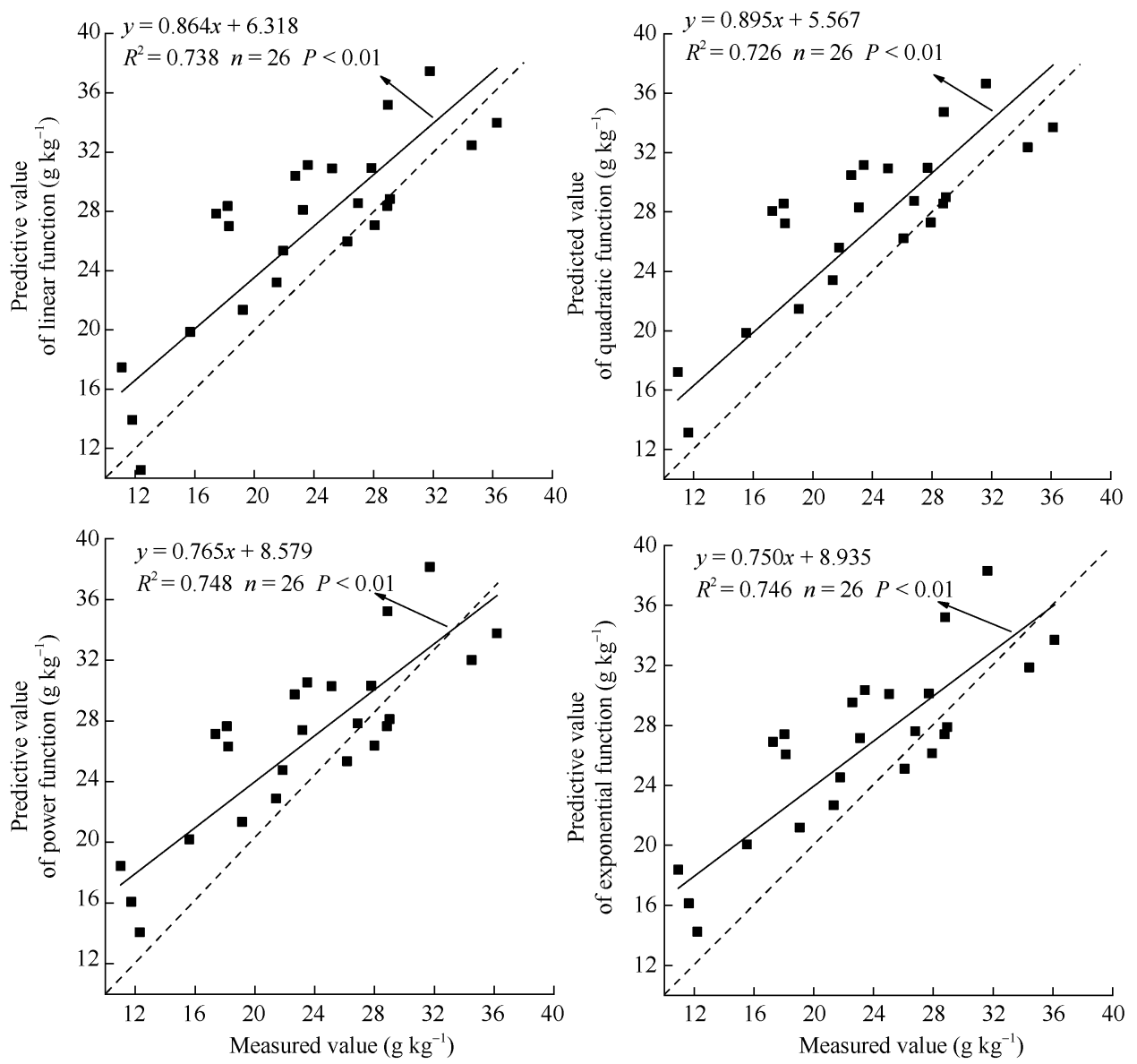

图 4 甜菜光谱特征参数 Dr762 (一阶微分最大正相关)下全氮含量估测模型验证

Fig. 4 Verification of the total nitrogen content estimation model under the spectral characteristic parameters Dr762 (maximum positive correlation of first order differential) of sugar beet

表 5 不同植被指数与全氮含量回归关系模型及验证

Table 5 Regression model and verification of different vegetation indexes and total nitrogen content

\begin{tabular}{|c|c|c|c|c|}
\hline \multirow{2}{*}{$\begin{array}{c}\text { 高光谱特征参数 } \\
\text { Characteristic parameter }\end{array}$} & \multicolumn{2}{|c|}{ 拟合模型 Fitting model } & \multicolumn{2}{|c|}{ 验证模型 Performance model } \\
\hline & 拟合方程 Equation & $R^{2}$ & $\operatorname{RE}(\%)$ & RMSE \\
\hline \multirow[t]{4}{*}{ SAVI } & $y=114.7 x-105.7$ & 0.541 & 24.492 & 5.092 \\
\hline & $y=104.5 x^{2}-122.1 x+28.18$ & 0.544 & 23.153 & 4.926 \\
\hline & $y=12.39 x^{5.208}$ & 0.539 & 21.878 & 4.830 \\
\hline & $y=0.1489 \mathrm{e}^{4.474 x}$ & 0.535 & 21.514 & 4.829 \\
\hline \multirow[t]{4}{*}{ Dr1138-Dr762 } & $y=49.36 x+0.4913$ & 0.732 & 19.708 & 4.709 \\
\hline & $y=-1.864 x^{2}+51.27 x+0.04145$ & 0.732 & 19.777 & 4.721 \\
\hline & $y=49.73 x^{0.9814}$ & 0.732 & 19.746 & 4.715 \\
\hline & $y=9.737 \mathrm{e}^{1.842 x}$ & 0.714 & 20.934 & 4.656 \\
\hline \multirow[t]{4}{*}{ Dr762-Dr496 } & $y=12.85 x+5.673$ & 0.794 & 23.008 & 5.372 \\
\hline & $y=-2.828 x^{2}+21.68 x-0.3212$ & 0.804 & 22.996 & 5.608 \\
\hline & $y=18.64 x^{0.755}$ & 0.799 & 23.025 & 5.447 \\
\hline & $y=11.91 \mathrm{e}^{0.4736 x}$ & 0.759 & 24.623 & 5.288 \\
\hline
\end{tabular}

$R^{2}$ : 决定系数; RE $(\%)$ : 相对误差; RMSE: 均方根误差。缩写同表 2 和表 3 。

$R^{2}$ : coefficient of determination; RE $(\%)$ : relative error; RMSE: relative root mean-squared error. Abbreviations are the same as those given in Table 2 and Table 3. 

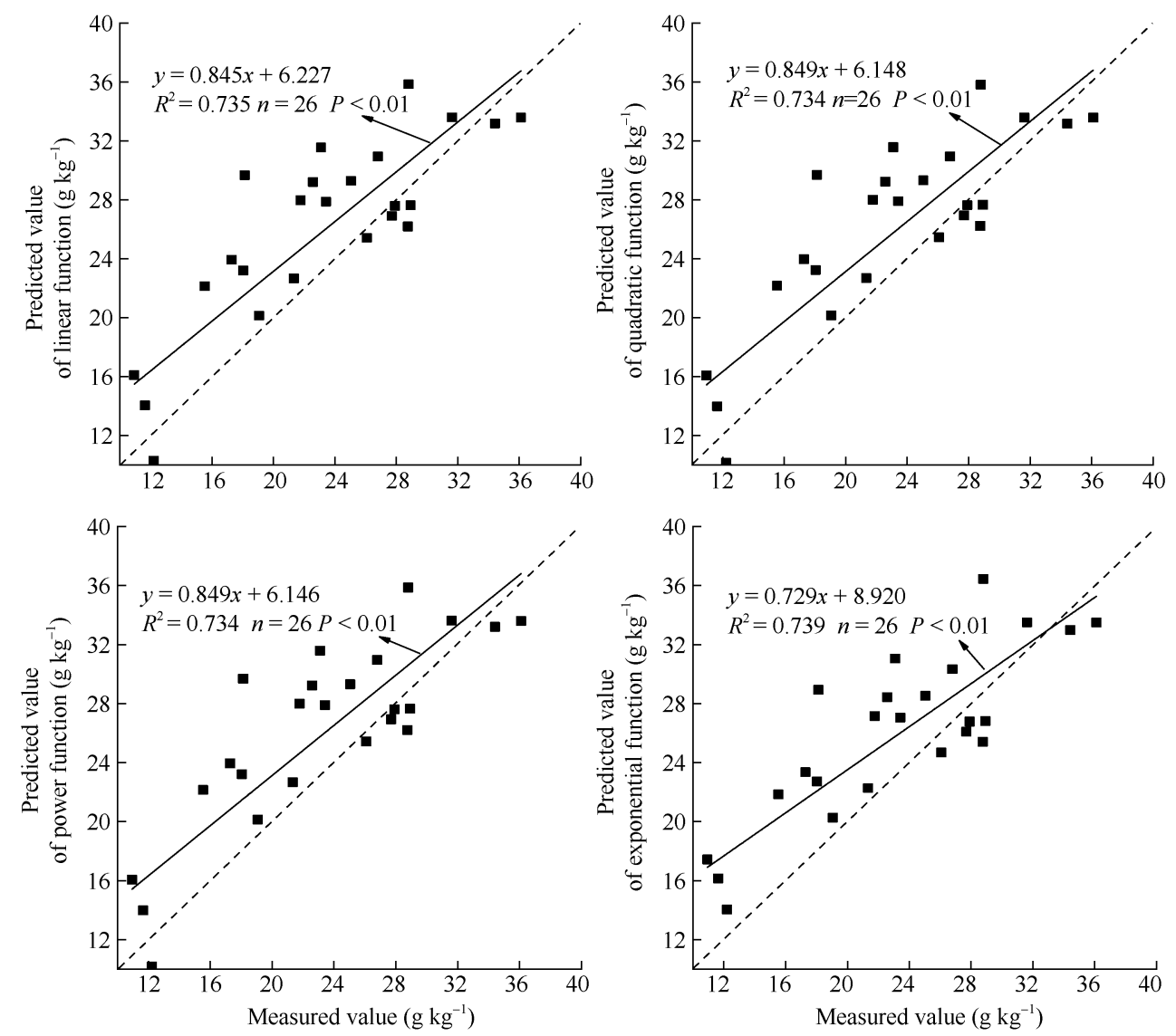

图 5 甜菜植被指数 Dr1138-Dr762 下全氮含量估测模型验证

Fig. 5 Validation of the estimation model of total nitrogen content under vegetation index Dr1138-Dr762

\section{3 讨论}

\section{1 不同氮素水平对甜菜全氮含量及冠层光谱} 反射率影响

在不同氮素水平下甜菜原始冠层光谱反射率波 形相似, 在 350 670 nm 波段范围内有明显的反射峰 与吸收谷, 光谱呈现先增高后降低的趋势, 在波段 670 760 nm 波形变化较为剧烈, 波形呈现为陡然上 升趋势, 在近红外波段 780 1300 nm 处形成近红外 反射平台, 且具有明显波形变化, 这与前人研究基 本一致 ${ }^{[37-38]}$ 。施氮处理 N75、N150、N225 在 $550 \mathrm{~nm}$ 较 CK 分别降低了 $7.5 \% 、 28.0 \% 、 23.0 \%$, 说明各施 氮处理相对 CK 均可提高甜菜植株冠层叶片对红、 蓝光的吸收, 提高植株的光合效率, 且施氮处理 N150 比 N75 与 N225 具有更好的提高植株光合效率 的作用; 在一阶导数光谱图中不同施氮处理除 N75 红边位置与 N0 相同, 其他施氮处理均出现“红移”现 象, 施氮处理 N150、N225 较 N0 均向长波方向移动 $5 \mathrm{~nm}$, 说明随着施氮量的增加, 甜菜植株冠层单位 光合效率有所提高。
3.2 光谱特征参数及植被指数的选择与全氮含 量的构建与检验

针对建立光谱特征参数及敏感植被指数进行篮 选，其中“三边”参数中红边面积具有较好估算全氮 含量的能力，在植被指数中 R767/R604、Dr1138Dr762 与 Dr762-Dr496 的相关性均在 0.6 以上, 其中 一阶微分光谱植被指数和红边光谱植被指数与叶片 全氮含量的相关性整体大于原始光谱植被指数的相 关性, 组合波段相对单一光谱特征参数相关性均有 所提高。

通过对光谱特征参数篮选建立全氮含量的多种 函数估算模型发现，不同光谱特征参数所建立模型 均表现为二次函数拟合效果最好, 利用检验样本对 所建立估测模型预测精度进行检验发现，虽然光谱 特征参数 $\operatorname{Dr} 762$ 二次函数估测模型决定系数具有最 大决定系数 $\left(R^{2}=0.772\right)$, 但验证模型中 $\mathrm{RE}(\%)$ 与 RMSE 较高, 通过对 Dr762 估测值与真实值拟合后 发现, 光谱特征参数 Dr762 幂函数下估算模型具有 更好的拟合优度。 

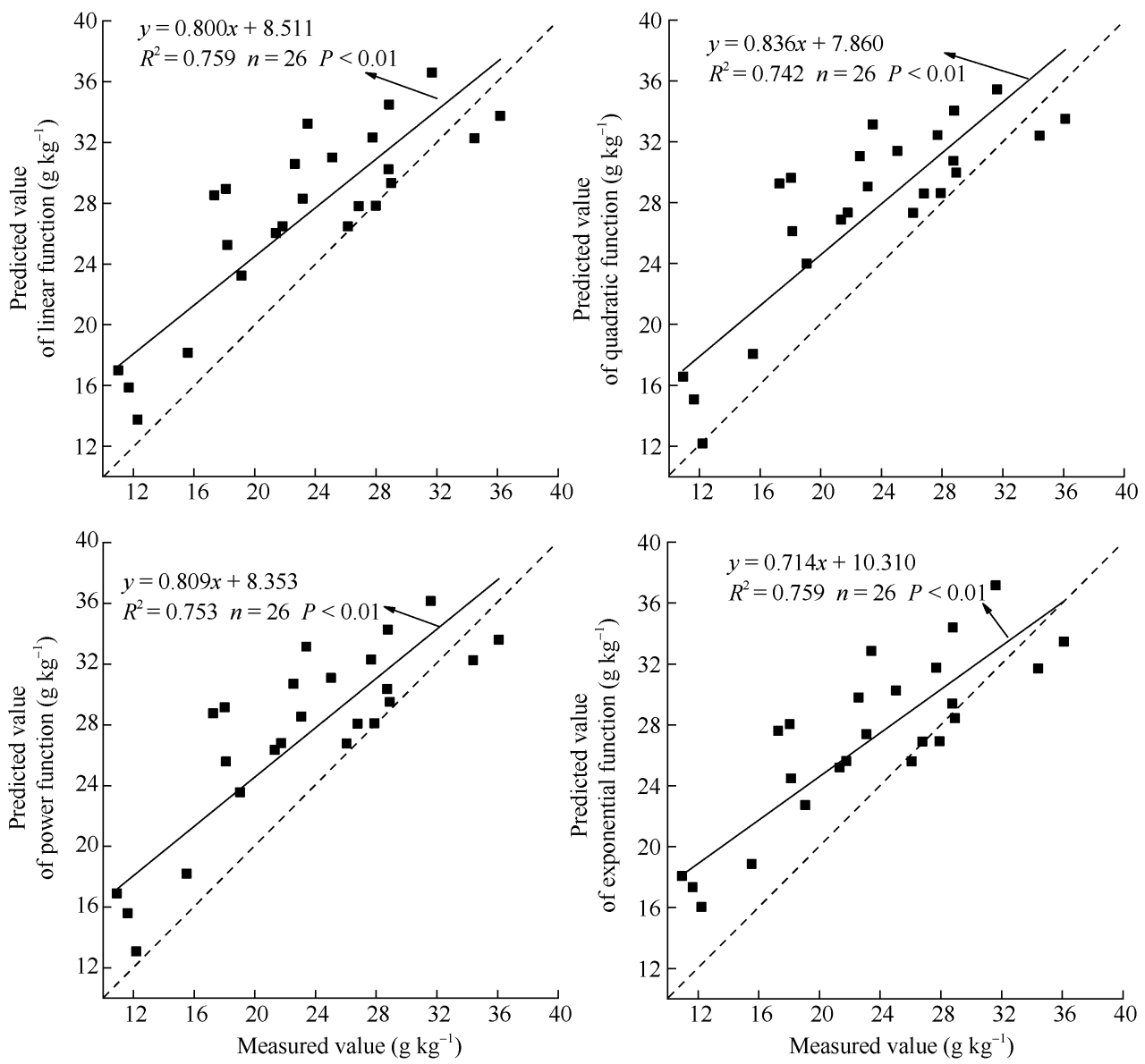

图 6 甜菜植被指数 Dr762-Dr496 下全氮含量估测模型验证

Fig. 6 Validation of the estimation model of total nitrogen content under vegetation index Dr762-Dr496

通过对敏感植被指数篮选建立全氮含量的多种 函数估算模型发现，不同植被指数所建模型均表现 为二次函数估算模型最优, 其中差值植被指数 Dr762-Dr496 下二次项估算模型的决定系数达到最 高值 $\left(R^{2}=0.804\right)$, 这说明在短波近红外波段与红光 波段组合和红光波段与绿光波段组合具有较好估算 叶片全氮含量的能力。利用检验样本对所建模型进 行检验发现, 虽然差值植被指数 Dr762-Dr496 表现 出较高拟合优度, 但验证模型中偏离真值的程度较 大，通过建立 Dr762-Dr496 估测值与真实值拟合后 发现, 差值植被指数 Dr762-Dr496 下一元线性函数 具有更好的拟合优度。

作物冠层叶片氮素含量的高光谱遥感估算较为 复杂, 受到作物冠层结构、叶片表面以及内部特征、 化学和光学特性、土壤背景值等一系列影响。 Knyazikhin 等 ${ }^{[39]}$ 研究认为冠层结构及其光学特性是 决定光谱反射率的主要因素, 冠层结构组织具有复 杂性, 光子在此结构中会产生碰撞、吸收、散射及
反射，只有一小部分会被截获，因而叶片反射率与 叶片氮素含量间无直接相关性。但叶片、冠层以及 生态系统之间的复杂联系可能会导致叶片全氮含量 与光谱反射率之间的重复相关性, 虽然可以确定冠 层光谱反射率的物理驱动因素是必要的, 模拟冠层 中作物生化指标可以起到较好作用，然而这些物理 机制在冠层、叶片内部结构及外部结构等和生态系 统之间的一系列生态驱动是联动运作的, 而氮素是 作物碳氮循环中一最为重要成分, 氮素有效性会较 大程度决定植被冠层丰度 ${ }^{[40-41]}$ 。碳氮循环作为生态 系统中运作的关键, 光合作用为植被提供生长及繁 殖的能量及物质, 其冠层氮素含量的定量变化提供 了关于植被冠层的直接信息 ${ }^{[42]}$ 。这也说明氮素含量 与冠层结构之间的联动, 并较大程度决定冠层结构, 并与冠层光谱反射率呈现直接或间接的影响。因此 对作物的生物学和物理学特性不能孤立地评估, 应 更好理解光谱处理方法以及工作原理，在更多波长 范围内进行更广泛的模拟。 
本文光谱数据测定主要在甜菜叶丛快速生长期 至糖分积累期, 在此阶段范围内甜菜冠层基本达到 土壤背景全覆盖, 通过对比表明前人建立植被指数 与本文全氮含量均具有较好相关性, 均达到极显著 相关，说明不同纬度地区、不同作物与所建立植被 指数均可进行全氮含量的估算, 但由于地区与作物 的不同, 所建立植被指数与叶片全氮含量的吻合度 存在一定的差异。本研究主要基于石河子滴灌甜菜 叶片全氮含量估算得到的初步结果, 但在实际工作 中利用高光谱遥感数据对甜菜叶片全氮含量估算时, 易受不同纬度地区以及冠层结构、叶片角度、结构 等因素影响, 因此所得结果还有待进一步验证。

\section{4 结论}

通过光谱特征参数与叶片全氮含量建立多种函 数估算模型, 其中光谱特征参数 Dr762 幂函数下估 算模型具有较好估算甜菜叶片全氮含量的能力, 其 决定系数 $R^{2}=0.747$, 验证相对误差 $\mathrm{RE}(\%)$ 为 21.635 , 验证均方根误差 RMSE 为 4.914。通过植被指数与叶 片全氮含量建立多种函数估测模型, 其中差值植被 指数 Dr762-Dr496 下一元线性函数具有较好估算甜 菜叶片全氮含量的能力, 其决定系数 $R^{2}=0.794$, 验 证相对误差 $\mathrm{RE}(\%)$ 为 23.008 , 验证均方根误差为 5.372 。

\section{References}

[1] Lee Y J, Yang C M, Chang K W, Shen Y. A simple spectral index using reflectance of $735 \mathrm{~nm}$ to assess nitrogen status of rice canopy. Agron J, 2008, 100: 205-212.

[2] 苏继霞, 王开勇, 费聪, 李阳阳, 樊华. 氮肥运筹对滴灌甜菜 产量、氮素吸收和氮素平衡的影响.土壤通报，2016，47: 1404-1407.

Su J X, Wang K Y, Fei C, Li Y Y, Fan H. Effects of nitrogen management on sugar beet yield, Nitrogen uptake and soil nitrogen balance under drip irrigation. Chin J Soil Sci, 2016, 47: 1404-1407 (in Chinese with English abstract).

[3] 费聪, 王维成, 李阳阳, 樊华. 氮素运筹对滴灌甜菜叶片光合 特性的影响. 江苏农业科学, 2016, 44(12): 227-229.

Fei C, Wang W C, Li Y Y, Fan H. Effects of nitrogen management on photosynthetic characteristics of sugarbeet leaves under drip irrigation. Jiangsu Agric Sci, 2016, 44(12): 227-229 (in Chinese).

[4] 高雨茜. 夏玉米叶绿素、叶面积指数高光谱估测研究. 西北农 林科技大学硕士学位论文, 陕西杨凌, 2016.

Gao Y Q. Chlorophyll and Leaf Area Indexstimation Based on Hyperspectrum of Summer Corn. MS Thesis of Northwest A\&F University, Yangling, Shaanxi, China, 2016 (in Chinese with English abstract).

[5] 刘冰峰. 夏玉米不同生育时期生理生态参数的高光谱遥感监
测模型. 西北农林科技大学博士学位论文, 陕西杨凌, 2016 .

Liu B F. Monitoring Models of Physiological and Ecological Parameters of Summer Maize Based on Hyperspectral Remote Sensing at Different Growth Stages. PhD Dissertation of Northwest A\&F University, Yangling, Shaanxi, China, 2016 (in Chinese with English abstract).

[6] Shibayama M, Akiyama T. Seasonal visible, near-infra-red and mid-infrared spectra of rice canopies in relation to LAI and above-ground dry biomass. Remote Sense Environ, 1989, 27: 119-127.

[7] Smith M L, Ollinger S V, Martin M E, Aber J D, Hallett R A, Goodale C L. Direct estimation of aboveground forest productivity through hyperspectral remote sensing of canopy nitrogen. Ecol Appl, 2002, 12: 1286-1302.

[8] Stone M L, Soile J B, Raun R. Use of spectral radiance for correcting in-season fertilizer nitrogen deficiencies in winter wheat. Trans ASAE, 1996, 39: 1623-1631.

[9] Thomas J R, Oerther G F. Estimating nitrogen content of sweet pepper leaves by reflectance measurements. Agron J, 1972, 64: 11-13.

[10] Tian Y C, Yao X, Yang J, Cao W X, Hannaway D B, Zhu Y. Assessing newly developed and published vegetation indices for estimating rice leaf nitrogen concentration with ground and spacebased hyperspectral reflectance. Field Crops Res, 2011, 120: 299-310.

[11] Wang W, Yao X, Yao X F, Tian Y C, Liu X J, Ni J, Cao W X, Zhu Y. Estimating leaf nitrogen concentration with three-band vegetation indices in rice and wheat. Field Crops Res, 2012, 129: 90-98.

[12] Menesatti P, Antonucci F, Pallottino F, Roccuzzo M, Allegra M, Stagno F, Intrigliolo F. Estimation of plant nutritional status by Vis-NIR spectrophotometric analysis on orange leaves. Biosyst Eng, 2010, 105: 448-454.

[13] Zhang G C, Li Z, Yan X M, Cheng C G, Zhou P, Lin G L, Zhou C J, Liu N, Han X R. Rapid analysis of apple leaf nitrogen using near infrared spectroscopy and multiple linear regression. Commun Soil Sci Plant Anal, 2012, 43: 1768-1772.

[14] 张洂元, 张立福, 张霞, 王树东, 田静国, 翟涌光. 不同光谱 植被指数反演冬小麦叶氮含量的敏感性研究. 中国农业科学, 2017, 50: 474-485.

Zhang X Y, Zhang L F, Zhang X, Wang S D, Tian J G, Zhai Y G. Sensitivity of different spectral vegetation index for estimating winter wheat leaf nitrogen. Sci Agric Sin, 2017, 50: 474-485 (in Chinese with English abstract).

[15] 王仁红, 宋晓宇, 李振海, 杨贵军, 郭文善, 谭昌伟, 陈立平. 基于高光谱冬小麦氮素营养指数估测. 农业工程学报, 2014, 30(19): 191-198.

Wang R H, Song X Y, Li Z H, Yang G J, Guo W S, Tan C W, Chen L P. Estimation of winter wheat nitrogen nutrition index using hyperspectral remote sensing. Trans CSAE, 2014, 30(19): 191-198 (in Chinese with English abstract).

[16] 田永超, 杨杰, 姚霞, 朱艳, 曹卫星. 利用红边面积形状参数 估测水稻叶层氮浓度. 植物生态学报, 2009, 33: 791-801.

Tian Y C, Yang J, Yao X, Zhu Y, Cao W X. Estimation of leaf canopy nitrogen concentration with red edge area shape parameter in rice. Chin J Plant Ecol, 2009, 33: 791-801 (in Chinese with 
English abstract).

[17] 顾清, 邓劲松, 陆超, 石媛媛, 王珂, 沈掌门. 基于光谱和形 状特征的水稻扫描叶片氮素营养诊断. 农业机械学报, 2012, 43(8): 170-174.

Gu Q, Deng J X, Lu C, Shi Y Y, Wang K, Shen Z M. Diagnosis of rice nitrogen nutrition based on spectral and shape characteristics of scanning leaves. Trans CSAM, 2012, 43(8): 170-174 (in Chinese with English abstract).

[18] 黄春燕, 王登伟, 问杰, 张煜星, 曹连莆, 程诚. 棉花叶绿素 密度和叶片氮积累量的高光谱监测研究. 作物学报, 2007, 33: 931-936.

Huang C Y, Wang D W, Yan J, Zhang Y X, Cao L P, Cheng C. Monitoring of cotton canopy chlorophyll density and leaf nitrogen accumulation status by using hyperspectral data. Acta Agron Sin, 2007, 33: 931-936 (in Chinese with English abstract).

[19] 吴华兵, 朱艳, 田永超, 姚霞, 刘晓军, 周治国, 曹卫星. 棉花 冠层高光谱参数与叶片氮含量的定量关系. 植物生态学报, 2007, 31: 903-909.

Wu H B, Zhu Y, Tian Y C, Yao X, Liu X J, Zhou Z G, Cao W X. Relationship between canopy hyperspectra parameter and leaf nitrogen concentration in cotton. Chin J Plant Ecol, 2007, 31: 903-909 (in Chinese with English abstract).

[20] 赵春江, 黄文江, 王纪华, 杨敏华, 薛绪掌. 不同品种、肥水条 件下冬小麦光谱红边参数研究. 中国农业科学, 2002, 35: 980-987.

Zhao C J, Huang W J, Wang J H, Yang M H, Xue X Z. Studies on the red edge parameters of spectrum in winter wheat under different varieties, Fertilizer and water treatments. Sci Agric Sin, 2002, 35: 980-987 (in Chinese with English abstract).

[21] 王树文, 赵越, 王丽凤, 王润涛, 宋玉柱, 张长利, 苏中滨. 基 于高光谱的寒地水稻叶片氮素含量预测. 农业工程学报, 2016, 32(20): 187-194.

Wang S W, Zhao Y, Wang L F, Wang R T, Song Y Z, Zhang C L, $\mathrm{Su} Z \mathrm{~B}$. Prediction for nitrogen content of rice leaves in cold region based on hyperspectrum. Trans CSAE, 2016, 32(20): 187-194 (in Chinese with English abstract).

[22] 陈兵, 王方永, 韩焕勇, 刘政, 邓福军, 林海, 余渝, 李少昆, 王克如, 肖春华. 基于光谱红边参数的棉花黄萎病叶片氮素 含量诊断研究. 棉花学报, 2013, 25: 254-261.

Chen B, Wang F Y, Han H Y, Liu Z, Deng F J, Lin H, Yu Y, Li S $\mathrm{K}$, Wang K R, Xiao C H. Monitoring nitrogen contents in leaves of cotton under verticillium wilt stress based on spectra red-edge parameters. Cotton Sci, 2013, 25: 254-261 (in Chinese with English abstract).

[23] Yao X, Zhu Y, Tian Y C, Cao W X. Exploring hyperspectral bands and estimation indices for leaf nitrogen accumulation in wheat. Int J Appl Earth Obs, 2010, 12: 89-100.

[24] Rouse J W, Haas R H, Schell J A, Deering D W. Monitoring vegetation systems in the Great Plains with ERTS. NASA SP, 1974, 351: 309.

[25] 梁亮, 杨敏华, 邓凯东, 张连蓬, 林卉, 刘志霄. 一种估测 小麦冠层氮含量的新高光谱指数. 生态学报, 2011, 31: 6594-6605.

Liang L, Yang M H, Deng K D, Zhang L P, Lin H, Liu Z X. A new hyperspectral index for the estimation of nitrogen contents of wheat canopy. Acta Ecol Sin, 2011, 31: 6594-6605 (in
Chinese with English abstract).

[26] Gitelson A A, Merzlyak M N. Spectral reflectance changes associated with autumn senescence of Aesculus hippocastanum L. and Acer platanoides L. leaves. Spectral features and relation to chlorophyll estimation. J Plant Physiol, 1994, 143: 286-292.

[27] Daniel A S, John A G. Relationships between leaf pigment content and spectral reflectance across a wide range of species, leaf structures and developmental stages. Remote Sense Environ, 2008, 81: 337-354.

[28] Gitelson A A, Merzlyak M N. Signature analysis of leaf reflectance spectra: algorithm development for remote sensing of chlorophyll. J Plant Physiol, 1996, 148: 494-500.

[29] Huete A R. A soil-adjusted vegetation index (SAVI). Remote Sense Environ, 1988, 25: 295-309.

[30] Rondeaux G, Steven M, Baret F. Optimization of soil-adjusted vegetation indices. Remote Sense Environ, 1996, 55: 95-107.

[31] Haboudane D, Miller J R, Pattey E, Zarco P J, Strachan I B. Hyperspectral vegetation indices and novel algorithms for predicting green LAI of crop canopy: monitoring and validation in the context of precision agriculture. Remote Sense Environ, 2004, 90: 337-352.

[32] Chen P F, Haboudane D, Trembley N, Wang J H, Vigneault P, Li B G. New spectral indicator assessing the efficiency of crop nitrogen treatment in corn and wheat, Remote Sense Environ, 2010, 114: 1987-1997.

[33] Gitelson A A, Vina A, Ciganda V, Rundquist D C, Arkebauer T J. Remote estimation of canopy chlorophyll content in crops. Geophys Res Lett, 2005, 32: 1-4.

[34] Serrano L, Penuelas J, Ustin S L. Remote sensing of nitrogen and lignin in Mediterranean vegetation decomposing biochemical from structural signals. Remote Sense Environ, 2002, 81: 355-364.

[35] Broge N H, Leblanc E. Comparing prediction power and stability of broadband and hyperspectral vegetation indices for estimation of green leaf area index and canopy chlorophyll density. Remote Sense Environ, 2001, 76: 156-172.

[36] 谌俊旭, 黄山, 范元芳, 王锐, 刘沁林, 杨文钰, 杨峰. 单作套 作大豆叶片氮素积累与光谱特征. 作物学报, 2017, 43: 1835-1844.

Chen J X, Huang S, Fan Y F, Wang R, Liu Q L, Yang W Y, Yang F. Remote detection of canopy leaf nitrogen status in soybean by hyperspectral data under monoculture and intercropping systems. Acta Agron Sin, 2017, 43: 1835-1844 (in Chinese with English abstract).

[37] 杨福芹, 冯海宽, 李振海, 杨贵军, 戴华阳. 基于可见光-近红 外光谱特征参数的苹果叶片氮含量预测. 农业机械学报, 2017, 48(9): 143-151.

Yang F Q, Feng H K, Li Z H, Yang G J, Dai H Y. Prediction for nitrogen content of apple leaves using spectral features parameters from visible and near infrared lights. Trans CSAM, 2017, 48(9): 143-151 (in Chinese with English abstract).

[38] 徐道青, 刘小玲, 王维, 陈敏, 阙画春, 李常凤, 郑曙峰. 淹水 胁迫下棉花叶片高光谱特征及叶绿素含量估算模型. 应用生 态学报, 2017, 28: 3289-3296. 
Xu D Q, Liu X L, Wang W, Chen M, Kan H C, Li C F, Zheng S F. Hyper-spectral characteristics and estimation model of leaf chlorophyll content in cotton under waterlogging stres. Chin J Appl Ecol, 2017, 28: 3289-3296 (in Chinese with English abstract).

[39] Knyazikhin Y, Mitchell A, Schull, Stenberg P, Mottus M, Rautiaien M, Yang Y, Marshak A, Carmona P L, Kaufmann R K, Lewis P, Disney M I, Vanderbilt V, Davis A B, Baret F, Jacquemoud S J, Lyapustin A, Myneni R B. Hyperspectral remote sensing of foliar nitrogen content. Proc Natl Acad Sci USA, 2013, 110: E185-E192.
[40] Ollinger S V, Reich P B, Frolking S, Lepine L C, Hollinger D Y, Richardson A D. Nitrogen cycling, forest canopy reflectance, and emergent properties of ecosystems. Proc Natl Acad Sci USA, 2013, 110: E2437.

[41] Townsen P A, Serbin S P, Kruger E L, Gamon J A. Disentangling the contribution of biological and physical properties of leaves and canopies in imaging spectroscopy data. Proc Natl Acad Sci USA, 2013, 110: E1704.

[42] Ustin S L. Remote sensing of canopy chemistry. Proc Natl Acad Sci USA, 2013, 110: 804-805. 